\title{
FACTORS IN THE DEVELOPMENT OF THE SPINAL STENOSIS SYNDROME
}

\author{
ARTHUR NAYLOR \\ From the Bradford Royal Infirmary
}

\begin{abstract}
The spinal stenosis syndrome is a potential hazard when congenital or developmental narrowing of the bony canal, particularly in its lateral recesses where it can be demonstrated by axial tomography, places the emergent nerve root and its blood supply at risk to further small compressive elements. When lumbar disc degeneration allows rotatory and lateral instability, posterolateral bulging of the annulus fibrosus into the root canal occurs when weight is taken on the ipsilateral lower limb. Symptoms of the spinal stenosis syndrome do not arise until the development of this instability. To relieve the symptoms, the nerve root must be freely mobilised and decompressed by full lateral decompression, with partial or total facetectomy if necessary, by enucleation of the intervertebral disc and by removal of the posterolateral portions of the annuli fibrosi.
\end{abstract}

The term spinal stenosis has tended to emphasise the role of deficiency in the size of the lumbosacral canal to the exclusion of the static and dynamic effects of soft-tissue changes in the canal and intervertebral disc.

\section{RELATIVE IMPORTANCE OF SIZE OF THE ROOT CANAL AND OF THE SPINAL CANAL}

The normal lumbosacral canal is narrowest in its anteroposterior diameter at the third and fourth lumbar vertebrae and its size increases caudally, but it tends to become more trefoil in shape at L5-S1. In women it tends to flatten in the lower part of the sacral region. As the canal progressively enlarges, the dural sac becomes reduced in volume and a myelogram is a less sensitive indicator of abnormalities of the lumbar contour and extradural contents in the lumbosacral region than in the middle and upper lumbar regions where the epidural space is larger. In the stenotic spine the facet joints, which are at the level of the intervertebral disc, are located more anteriorly and medially in association with short pedicles, the intervertebral foramina being narrowed.

Measurements of the bony contour of the lumbar spine, particularly the anteroposterior diameters, have been made on plain radiographs or at operation by means of a stenosiometer (Sarpyener 1945); recorded results range from 12 millimetres (Verbiest 1954, 1975) to 14 millimetres (Huizinger, Van der Heiden and Vinken 1952) and 15 millimetres (Epstein, Epstein and Levine 1962). This measurement is purely an indication of potential for the development of stenotic symptoms; it does not reveal the essential site of the stenosis which is producing the symptoms. The important measure- ment is that of the root canal, which cannot be shown on plain radiographs, but can be well demonstrated by computerised axial tomography or by transverse axial tomography. The latter is effected with the patient supine with hips and knees flexed to obliterate the lumbar lordosis, and with the $\mathrm{x}$-ray tube tilted to 20 degrees with the horizontal and rotating through an arc of 220 degrees at a standard distance from the patient (Jacobson, Gargano and Rosomoff 1975; Sheldon, Russin and Gargano 1976).

An axial tomogram at the level of the L5-S1 intervertebral disc shows the dorsolateral wall of the spinal canal formed by the L5 lamina and superior facet of the sacrum, while on the ventrolateral aspect the superior facet of the sacrum and the inferior facet of the fifth lumbar vertebra form the entrance to the root canal (Fig. 1). Enlargement of one or both articular facets narrows the lateral recess still further. The thickened laminae and facets not only compress the roots leaving the canal at that segment but also involve roots within the theca at the same level (Fig. 2). The nerve root in the narrowed lateral recess is liable to further compression by additional factors in the form of a disc herniation (Fig. 3 ), or bulging of an incompetent annulus fibrosus.

Reduced space in the lumbosacral canal or dural sac does not necessarily produce the syndrome, since marked narrowing of the dural canal has been noted in surgical shock when there are no stenotic symptoms. The space can be functionally nil, as when the theca is filled with tumour (Fig. 4), but if the lateral recesses are not obstructed significant symptoms will arise only when the dura has become stretched by the developing tumour.

Symptoms of stenosis may arise in a canal of normal

A. Naylor, Ch.M., M.Sc., F.R.C.S., Consultant in Orthopaedic and Accident Surgery, 3 Mornington Villas, Manningham Lane, Bradford, Yorkshire BD8 $7 \mathrm{HE}$, England. 


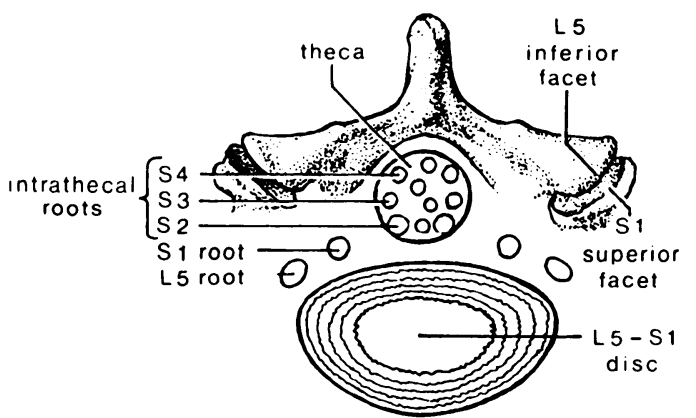

Fig. 1

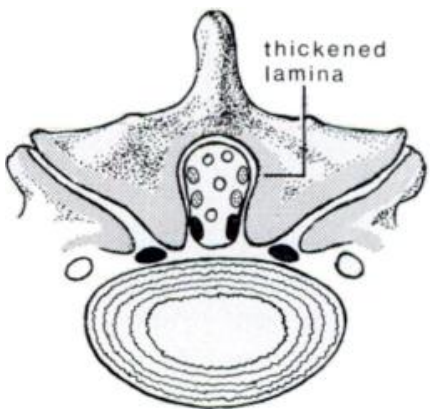

Fig. 2

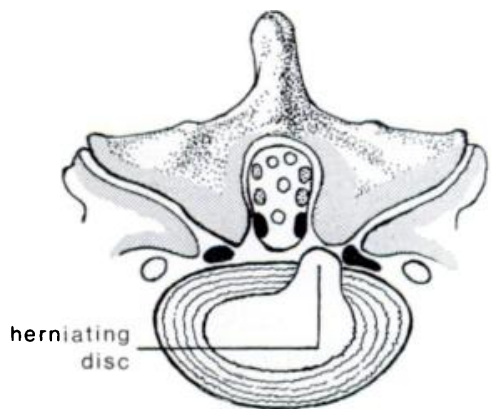

Fig. 3

Figure 1-Diagram representing an axial tomogram of the normal spine at level of the L5-S1 intervertebral disc. The fifth lumbar nerve root leaves the root canal and the first sacral root is closely related to the inferior facet of $\mathrm{L} 5$ as it leaves the dural sac. Figure $2-$ When stenosis is present, the articular facets and adjacent thickened laminae (hatched) reduce the coronal diameters of the spinal canal and narrow the lateral recesses, compressing the emergent root, $\mathrm{S} 1$, the theca (producing the typical waisting appearance) and the intrathecal roots. Disc bulging and instability during walking will precipitate symptoms from more than one root. Figure 3 - In the presence of stenosis, the emerging nerve root is more vulnerable to compression by a small lateral disc herniation. Enucleation must be combined with wide lateral decompression. Fenestration gives incomplete relief of intrathecal and extrathecal root involvement.

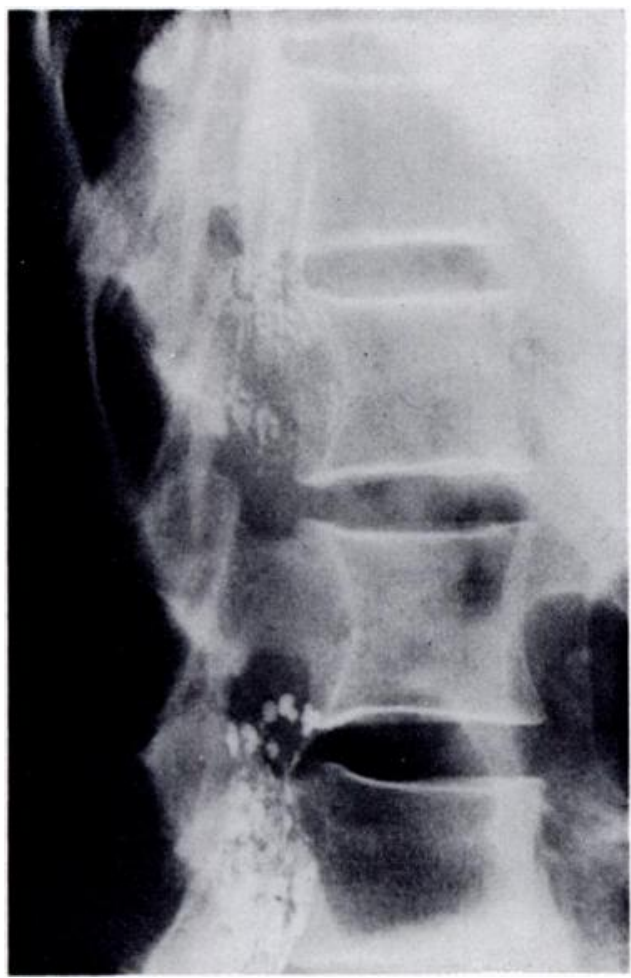

Fig. 4

Myelogram showing complete obliteration of the theca by a tumour of the cauda equina (ependymoma) with "scalloping" of the posterior aspects of the vertebral bodies; thus complete functional "stenosis" of the canal was present, but no stenotic symptoms were experienced.

size, but only if the nerve root is fixed by extrathecal adhesions which obliterate the canal. This is seen where a successful disc enucleation is followed by the later development of stenotic symptoms.

Case 1. This man aged fifty-two years, with a history of recurrent episodes of low backache and right sciatica for ten years, was first seen in an acute episode. Radiographs showed a large transverse process at
L5 and spondylitis of L3-S1 but no changes in the facet joints. As the many recurrences interfered with his normal occupation, disc enucleation was undertaken in October 1968. At operation a large lateral prolapse at L5-S1 was removed. His symptoms slowly resolved and he returned to work as a grinding engineer in February 1969. At that time he had some discomfort in the distribution of the first right sacral nerve when he walked 500 to 600 yards.

He was seen again in July 1973, aged fifty-seven, when the claudication distance was 30 yards. Pulses in the leg were normal. There was no muscle weakness, but the right ankle and knee jerks were depressed. Spinal flexion was markedly restricted and painful. Radiographs showed traction spurs at L5-S1 and unchanged spondylitis at L3-5. A radiculogram (Dimer X) showed no filling of the root sleeve of the first right sacral nerve. In October 1973 he underwent a second operation at which the first sacral root was found to be encased in fibrous tissue, completely obliterating the lateral recess. Neurolysis was undertaken together with the removal of the underlying portion of the annulus. This relieved his symptoms.

Stenotic symptoms can be produced even in the presence of a normal anteroposterior diameter when rotatory deformity of the vertebrae and facet asymmetry produce a unilateral narrowing of the lateral recess. Only axial tomography will show this adequately (Figs. 5 to 7). Spondylitic change in the facet joints will arise due to the abnormal stress placed on the asymmetrical joints and this will reduce the size of the root canal still further.

The large superior facet found in spondylitic stenosis causes the nerve root to take a longer and more angled course. This is evident in patients suffering from Paget's disease of the vertebrae, where increase in the diameters of the vertebral body narrows the spinal canal, the lateral recess and the intervertebral foramina. These combine to produce an angulation of the nerve root around the posterior articular facets, interfering with its blood supply.

The vascular arrangements of the nerve root are highly vulnerable to compression within the root of the canal and in the intervertebral foramen. Medullary and radicular arteries arise to supply both the intraspinal section of the nerve root and the root beyond the 
foramen (Dommisse 1974). The veins consist of complicated sinosoidal channels which are equally vulnerable to reduction of space in the root canal. In the experimental animal, exercise of a single limb can be shown to be accompanied by dilatation of the blood vessels on the emergent nerve root in the intervertebral foramen (Blau and Rushworth 1958). Compression within the lateral recess is liable to prevent their adequate dilatation to allow the additional blood flow necessary during walking. indicating that there is still considerable mobility and probably instability in the lumbar spine. Hirsch and Nachemson (1954) showed in the experimental model that, when an intervertebral disc is compressed on one side, bulging appears on the side of the compression. If an isolated disc preparation is loaded from 100 to 400 kilograms, bulging due to the compression normally causes a change in radius of 0.8 per cent. In the degenerate disc, however, the bulging is at least 30 per cent. In patients with stenotic symptoms annular bulging

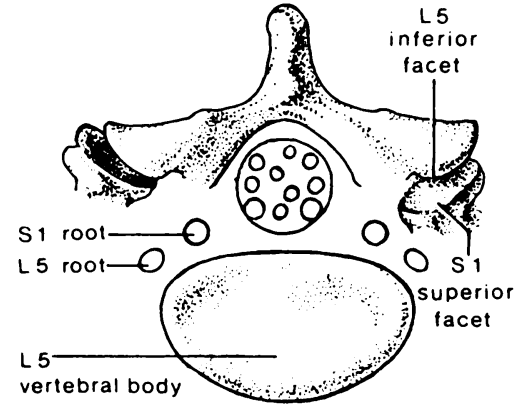

Fig. 5

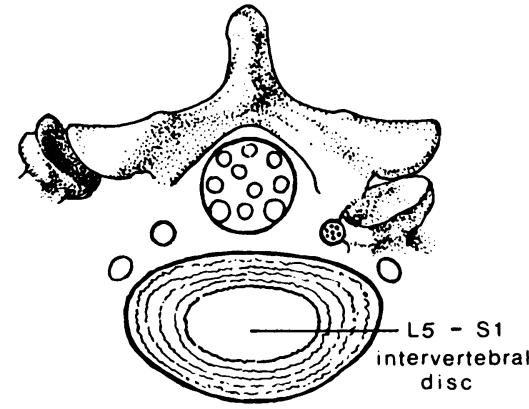

Fig. 6

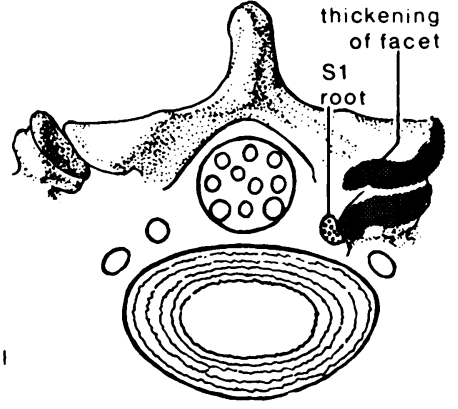

Fig. 7

Diagrams representing transverse axial tomograms showing the relationship of L5, S1 and S2 nerve roots to the asymmetrical left facet joint at the lumbosacral junction. The facet joint is at the level of the intervertebral disc and the spinal canal is narrowest at that point. Figure 5-A section through the lower border of L5. Figure $6-A$ section through the superior border of the L5-S1 intervertebral disc. At the lower border of the disc, the S1 root lies in the gutter between the disc and the superior facet. Figure 7-Spondylitic thickening of the facets (hatched) increases the vulnerability of the S1 root to compression.

Spinal extension is restricted and painful in the stenotic patients who usually walk or stand with the lumbar spine slightly flexed. When walking, the roots are tight on spinal flexion but on extension they slacken and their cross-section increases but the intervertebral foramen narrows. Thus it is probable that additional compression of the nerve root and blood vessels in the intervertebral foramen and nerve root canal produces stenotic symptoms during extension. On standing with a normal or exaggerated lumbar lordosis, especially if bony stenosis is already present, the foramen will be reduced in size. Reducing the lordosis opens up the foramen and reduces any interference with the circulation around the nerve root.

\section{SPINAL MOVEMENT AND INSTABILITY}

It is the thesis of this paper that the important precipitating factor in a patient who already has a bony stenosis, or an obliterated nerve root canal, is spinal movement and this produces its effect by means of the malfunction of the associated degenerate intervertebral disc and consequent inefficient weight distribution, resulting in bulging of the annulus alternately into each lateral recess. Gross lumbar spondylosis associated with spinal rigidity is not usually accompanied by the stenosis syndrome. In the stenotic patient, however, radiography often reveals only a moderate degree of spondylosis, of the incompetent intervertebral disc posterolaterally into the lateral recess and nerve root canal is seen on radiculography and at operation, giving a segmental waisting appearance and lack of filling of the root sleeve.

Inefficient distribution of the load by the degenerate disc, associated with alternate lateral compression during walking, results in anteroposterior, rotational and lateral instability of the segment and increased intermittent annular bulging on the side of compression, thus interfering with the vessels of the nerve root. Support for this view is afforded by the relief of stenotic symptoms by intervertebral fusion although the space in the spinal canal and lateral recesses is unaltered by the procedure. This can be well demonstrated in patients suffering from both lytic and degenerative types of spondylolisthesis. In the latter, developmental malrotation of the intervertebral body contributes to stenosis of the lateral recess.

Case 2. This man aged forty-seven years presented with a two-year history of low backache with claudication in the right leg, worse when going uphill. No neurological defect was noted, pulses in the lower limbs were normal, radiographs revealed spondylolisthesis with advanced degenerative changes at L5-S1 but there was no lytic lesion in the pars interarticularis. Intertransverse fusion was performed in October 1969, since when he has remained free of symptoms.

Case 3. This man aged forty-four years presented with a two-year history of pain on the outer side of the right calf and ankle and on the posterior aspects of the left thigh which occurred only when walking. Symptoms were markedly aggravated when going uphill, but he had no 
symptoms at rest. Examination revealed no reflex change or muscle wasting. Radiographs revealed degenerative spondylolisthesis at L5-S1. Intertransverse fusion of L4-S1 in December 1971 relieved the symptoms.

\section{ANALOGY OF LUMBAR SPINAL STENOSIS AND CERVICAL SPINAL MYELOPATHY}

One can draw a close analogy between cervical spinal myelopathy and the lumbar spinal stenosis syndrome. In both conditions there is a pre-existing stenotic canal, particularly in the lateral recesses. The anteroposterior diameter of the normal cervical spinal canal, from the base of the spinous process to the most posterior part of any osteophytic outgrowth, is 17 to 22 millimetres, but when it is 12 millimetres or less cervical spinal myelopathy is prone to develop (Adams and Logue 1971). A factor in both conditions is the vulnerability of the collateral arterial and venous supply to the spinal cord and nerve roots in the intervertebral foramen and root canals, this being further accentuated by spinal movement. In the cervical spine, flexion movement and instability due to disc degeneration interfere still further with the circulation of the spinal cord by causing direct compression of the anterior segment of the cord against the osteophytes in the stenosed canal. In the lumbar region, the movement of the spine on walking precipi- tates these symptoms because the instability of the degenerate disc results in bulging of the annulus into the lateral recess, thus compressing the nerve root which is already at risk in an extremely reduced space.

\section{CONCLUSIONS}

There are three factors essential to the production of the spinal stenosis syndrome. First, a basic congenital or developmental defect reduces the space in the lateral recess in the spinal canal. This may be present for many years without symptoms. Axial tomography is necessary to demonstrate the changes in the lateral recesses. Secondly, fixity of the nerve root together with a long. angular course round the larger arthritic facet joints interferes with vascularisation. Thirdly, symptoms arise on walking because of bulging of the annulus into the nerve root canal, and rotatory and lateral instability due to degeneration of the intervertebral disc.

To produce adequate relief of stenotic symptoms, simple bilateral laminectomy is inadequate. The nerve root canals on each side need to be decompressed, with partial or total facetectomy if necessary, to give mobility of the nerve root and restoration of the normal course. At the same time the degenerate disc, which can be seen bulging posterolaterally, needs enucleation together with removal of the bulging annulus in the lateral recess.

\section{REFERENCES}

Adams, C. B. T., and Logue, V. (1971) Studies in cervical spondylotic myelopathy, parts I, II and III. Brain, 94, 557-594.

Blau, J. N., and Rushworth, G. (1958) Observations on the blood vessels of the spinal cord and their responses to motor activity. Brain. 81, 354-363.

Dommisse, G. F. (1974) The blood supply of the spinal cord. Journal of Bone and Joint Surgery, 56-B, 225-235.

Epstein, J. A., Epstein, B. S., and Lavine, L. (1962) Nerve root compression associated with narrowing of the lumbar spinal canal. Journal of Neurology, Neurosurgery and Psychiatry, 25, 165-176.

Hirsch, C., and Nachemson, A. (1954) New observations on mechanical behavior of lumbar discs. Acta Orthopaedica Scandinavica, 23, 254-283.

Huizinga, J., Van der Heiden, J. A., Vinken, P. J. J. G. (1952) The human lumbar vertebral canal-a biometric study. Koninklijke Nederlandse Akadamie van Wetenschappen, Proceedings of the Section of Sciencts, C55, 22-33.

Jacobson, R. E., Gargano, F. P., and Rosomofi, H. L. (1975) Transverse axial tomography of the spine. Parts I and II. Journal of Neurosurgery. 42, 406-419.

Sarpyener, M. A. (1945) Congenital stricture of the spinal canal. Journal of Bone and Joint Surgery, 27, 70-79.

Sheldon, J. J., Russin, L. A., and Gargano, F. P. (1976) Lumbar spinal stenosis. Radiographic diagnosis with special reference to transverse axial tomography. Clinical Orthopaedics and Related Research, 115, 53-67.

Verbiest, H. (1954) A radicular syndrome from developmental narrowing of the lumbar vertebral canal. Journal of Bone and Joint Surgery. 36-B, 230-237.

Verbiest, H. (1975) Pathomorphologic aspects of developmental lumbar stenosis. Orthopedic Clinics of North America, 6, $177-196$. 